\title{
Care-seeking and treatment for sick children-Insights for improving malaria, family planning, and maternal and child health outcomes in northwestern Nigeria through social and behavior change programming
}

Breakthrough RESEARCH

Follow this and additional works at: https://knowledgecommons.popcouncil.org/departments_sbsr-rh How does access to this work benefit you? Let us know!

\section{Recommended Citation}

Breakthrough RESEARCH. 2020. "Care-seeking and treatment for sick children-Insights for improving malaria, family planning, and maternal and child health outcomes in northwestern Nigeria through social and behavior change programming," Programmatic Research Brief. Abuja: Population Council. 


\section{Care-seeking and treatment for sick children}

This brief provides rigorous evidence-based insights to implementers and researchers of social and behavior change (SBC) programs that seek to improve knowledge, attitudes, norms, and behaviors for caretakers of young children. The brief focuses on care-seeking and treatment behaviors for sick children. It summarizes the barriers and facilitators to seeking care from formal medical sources and receiving appropriate diagnosis or treatment for children under two years of age with symptoms of fever, diarrhea, or cough with fast or difficult breathing. It is one of a series of briefs that present findings from a Breakthrough RESEARCH study that uniquely captures data on a wide range of psychosocial drivers of behavioral outcomes in the areas of family planning, malaria, and maternal, newborn, and child health, and nutrition ( $\mathrm{MNCH}+\mathrm{N})$. The results presented in this series can inform the improvement of women and children's health programming in Nigeria and help to achieve the objectives of the National Strategic Health Development Plan II (2018-2022), ${ }^{1}$ as well as support global efforts to achieve the United Nations Sustainable Development Goals. ${ }^{2}$

\section{Breakthrough RESEARCH and Breakthrough ACTION in Nigeria}

Breakthrough RESEARCH and Breakthrough ACTION are USAID's flagship SBC programs. Breakthrough ACTION in Nigeria implements SBC programming in eleven states and the Federal Capital Territory (FCT) by targeting key psychosocial factors at multiple socio-ecological levels (e.g., individual, community, society) in order to improve health behaviors in the areas of malaria, family planning, and $\mathrm{MNCH}+\mathrm{N}$.

\section{KEY POINTS}

Only one-third of children with symptoms of fever, diarrhea, or cough with fast or difficult breathing were taken to a formal medical source. Only $21 \%$ of children with diarrhea received ORS and zinc, while only 22 percent of under-2s with fever were tested for malaria.

Among women who took their sick child to any facility, provider trust, nearby location, and perceived effectiveness of treatments were the main reasons for choosing the treatment location.

Women who took their sick child to a pharmacy more often cited short wait time, lower cost, and nearby location as reasons for going to this location. Women who attended government hospitals or PHCs more commonly cited provider trust and perceived effectiveness of treatments as drivers of their choice.

Increased awareness of pneumonia and diarrhea treatment is a critical first step for helping to improve the uptake of formal care-seeking and recommended treatment, alongside improving overall health services quality.

Women had strong doubts about the accuracy of malaria tests and worried that a fever could still be due to malaria even if the test result is negative. SBC programs can work to dispel doubts about negative malaria test results in conjunction with improving health services quality and malaria test availability.

Breakthrough RESEARCH in Nigeria conducts rigorous research to inform SBC program implementation in three of these program states (Kebbi, Sokoto, and Zamfara). Findings presented here are from a Breakthrough RESEARCH baseline 
study that will be useful for informing SBC program adaption and scale-up in Nigeria.

\section{Setting the context}

Pneumonia, diarrhea, and malaria are the leading causes of child mortality globally and in Nigeria. ${ }^{1}$ It is estimated that these causes combined are responsible for 42 percent of deaths among children under five years of age in Nigeria (14\% for malaria, $10 \%$ for diarrhea and $18 \%$ for pneumonia). A cornerstone of child survival programs is to ensure appropriate care-seeking, diagnosis/classification and treatment of these diseases according to the World Health Organization's Integrated Management of Childhood IIIness framework, ${ }^{2}$ which was updated in 2010 to include malaria diagnostic testing. ${ }^{3}$

Yet, care-seeking from formal medical sources and receipt of recommended treatment for sick children in Nigeria remains quite low. ${ }^{4}$ In 2018, only $23 \%$ of children under five years of age with diarrhea in the previous two weeks received oral rehydration solutions (ORS) and zinc treatment. Similarly, only 14 percent of under-5s with fever in the past two weeks received a malaria diagnostic test. ${ }^{4}$ Several studies indicate that low rates of seeking care from a formal medical source and use of recommended treatment in the northwestern region may be related to cost of services, facility distance, or lack of transportation, and poor awareness about common childhood illnesses. ${ }^{5-6}$ Other studies suggest that sociocultural norms and gender dynamics play an important role in care-seeking for childhood illnesses, given restrictions on women's movements in this region..$^{7-8}$ In a 2012 study, there was also an expressed distrust of health services linked to polio immunization campaigns resulted in negative impressions of immunizations and health services in the region. ${ }^{?}$

Decision-making processes are multifaceted and influenced by the complex interaction of knowledge, beliefs, social norms, and other psychosocial factors that are posited by theories as intermediate determinants of behavior change..$^{9-10}$ Based on previous research and theory-based design, we developed a set of ideational metrics for pediatric malaria, pneumonia, and diarrhea care-seeking behaviors for the northwestern Nigerian context. ${ }^{11-12}$ This research brief aims to describe different barriers and facilitators of care-seeking and treatment for sick children and to quantify the importance of ideational factors on key behavioral outcomes.

\section{Study methods}

Results are based on the Behavioral Sentinel Surveillance (BSS) baseline survey that was conducted between September and October 2019 in Breakthrough ACTION program areas in Kebbi, Sokoto, and Zamfara States of northwestern Nigeria. Figure 1 summarizes the study methods.

\section{FIGURE 1 BSS BASELINE SURVEY STUDY METHODS}

Study

population

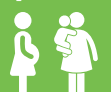

Pregnant women and women with under-2s areas in Kebbi, Sokoto andd Zamfara states (not representative at state level)

\section{Study}

design

(r)

$\Delta$

Cross-sectional and cohort components

Sample

size

לै

3,032 pregnant women

3,043 women with a child under 2 years

method

$\because 80$

Random selection of women with a child under 2 years

Data Mixed-effects logistic regression models were

analysis used to derive predicted probabilities for ANC4+ (four or more visits) and facility-based delivery, controlling for pregnancy-related ideations and sociodemographic characteristics, including household wealth, age, education (woman and spouse), and employment (woman and spouse). living within Breakthrough ACTION program

Sampling 108 wards across 3 states

Census of pregnant women

\section{Key Results}

\section{Low care-seeking, diagnosis, and treatment practices for sick children}

- Only about one-third of children under two years of age with fever, diarrhea, or respiratory symptoms (cough with difficult or rapid breaths) were taken to a formal medical source $^{a}(34 \%, 34 \%$ or $39 \%$, respectively) (Figure 2).

- Nearly half of children under-two years of age with diarrhea, respiratory symptoms or fever were taken to an informal service provider such as a pharmacy/chemist (47\%, 53\%, or $44 \%$ respectively).

- Less than one-quarter (22\%) of children under two years of age with fever in the past two weeks were tested for malaria. Among children under two years of age with diarrhea in the past two weeks, only 21 percent were given ORS and zinc treatment.

${ }^{a}$ Formal medical source refers to government hospital, government PHC, dispensary/health post, community health outreach post, nursing/maternity home, or private hospital/clinic. Informal sources refer to pharmacist/chemist, drug shop, traditional practitioner, family, or friend. 


\section{FIGURE 2 FORMAL CARE-SEEKING, DIAGNOSTIC TEST UPTAKE AND RECOMMENDED TREATMENT FOR SICK CHILDREN IS LOW}

Percentage of last-born children under two years of age with fever, diarrhea or respiratory symptoms (cough with fast or difficult breathing) taken to a formal medical source* and given the recommended diagnostic test or treatment

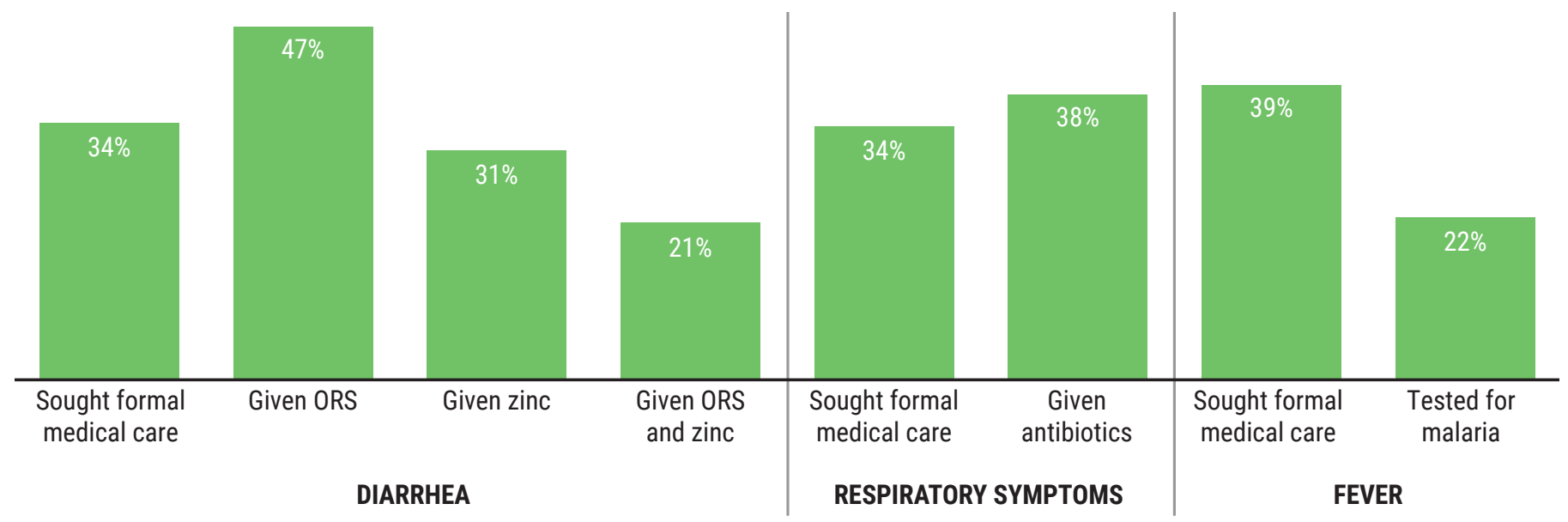

*Formal medical source refers to government hospital, government PHC, dispensary/health post, community health outreach post, nursing/maternity home, or private hospital/clinic. Informal sources refer to pharmacist/chemist, drug shop, traditional practitioner, family, or friend.

\section{Provider trust, nearby location, and perceived effective treatment were the main reasons for choosing treatment locations}

- Among respondents who did not seek care for their sick child, the reasons cited were similar for each illness symptom. The most commonly cited reasons were perceived healthcare costs, fatalistic attitudes ("Up to God"), and perceptions of non-severe symptoms or provision of home care. In contrast to other health areas (e.g., maternal health), few respondents cited spousal opposition as a main reason for not seeking care for a sick child (Table 1).

- Among respondents who sought care for their sick child, the reasons for choosing the treatment location were also similar across illness symptoms. The most commonly cited reasons were provider trust, nearby location, and perceived effectiveness of treatments prescribed. Nearby location, low cost, short wait time, or family/friend recommendation were more often cited among respondents attending pharmacies/chemists ${ }^{b}$ than government hospitals or PHCs. In contrast, provider trust, perceived effectiveness of treatments, and perceptions that they will receive respectful care were more often cited among respondents attending government hospitals or PHCs than pharmacies (Figure 3, next page).

\section{TABLE 1 COSTS, FATALISM AND NON-SEVERE SYMPTOMS ARE MAIN REASONS FOR NOT SEEKING CARE FOR SICK CHILDREN}

Among women 15-49 years with a child under two years of age who did not seek any care for the youngest child with illness symptoms in the past two weeks, percentage who cited certain reasons for not seeking care

\begin{tabular}{|lccc|}
\hline & DIARRHEA & $\begin{array}{c}\text { COUGH WITH } \\
\text { FAST OR } \\
\text { DIFFICULT } \\
\text { BREATHING }\end{array}$ & FEVER \\
\hline Too expensive & 32 & 33 & 31 \\
Fatalism (Up to God) & 29 & 15 & 33 \\
Non-severe symptoms/ & 25 & 25 & 21 \\
provided home care & & 10 & 10 \\
Spousal opposition & 3 & 1 & 3 \\
Facility distance & 6 & 9 & 1 \\
Facility closed & 3 & & \\
\hline
\end{tabular}




\section{Programmatic implications}

$\checkmark$ SBC programs, working together with health system strengthening efforts, should consider improving both perceived and actual health services quality to extend the availability and use of recommended treatments for sick children. These efforts should consider reasons that drive care seeking to informal sources, including short wait times, nearby locations, and low cost.

$\checkmark$ Perceived health care costs, in particular, were a main barrier to seeking any care and also drove care-seeking at pharmacies making this a potentially important focus for health system strengthening efforts.

$\checkmark$ SBC programs may also consider focusing on raising women's knowledge about recommended pneumonia and diarrhea treatment practices, addressing misperceptions about common childhood illnesses, and improving perceptions of health services quality.

\section{Malaria knowledge is high while diarrhea and pneumonia knowledge is low}

- Less than 20 percent knew that washing hands can prevent diarrhea and pneumonia. Only 28 percent knew that rapid or difficult breathing are key symptoms of pneumonia. Nearly half (43\%) had never heard of zinc or were able to say how it helps a child with diarrhea (Figure 4).
- For diarrhea, only 8 percent of women in the poorest households knew that handwashing helps prevent diarrhea compared with 30 percent in the wealthiest households. Similarly, 15 percent in the poorest households had never heard of zinc compared to 2 percent in the wealthiest ones. For pneumonia, 23 percent in the poorest households knew antibiotics were effective pneumonia treatment compared to 37 percent in wealthiest ones.

- Although 74 percent agreed that a blood test is the only way to know if a person has malaria, 61 percent said that, even if the test result is negative, they always worry that the fever could still be due to malaria. One-third of respondents strongly agreed with this statement indicating firmly held beliefs about the (in)accuracy of negative malaria test results.

\section{Programmatic implications}

$\checkmark$ SBC programs should consider focusing on raising awareness about childhood pneumonia and diarrhea among adults, particularly in the poorest households where knowledge is lowest. While malaria knowledge is generally high, SBC programs may need to focus on dispelling doubts in negative malaria test results.

\section{Perceptions about health service quality are critical for seeking formal care for sick children}

- Women who believed health facilities frequently have the medicines needed for sick children had 2.2-, 1.9-, and

\section{FIGURE 3 DIFFERENT REASONS DRIVE THE CHOICE OF GOVERNMENT HOSPITAL, PHC, OR PHARMACY FOR TREATING SICK CHILDREN}

Among women 15-49 years with a child under two years of age who sought any care for her youngest child with symptoms of fever, diarrhea, or cough with respiratory symptoms in the past two weeks, percentage who cited certain reasons for choosing the treatment location, by source of care visited

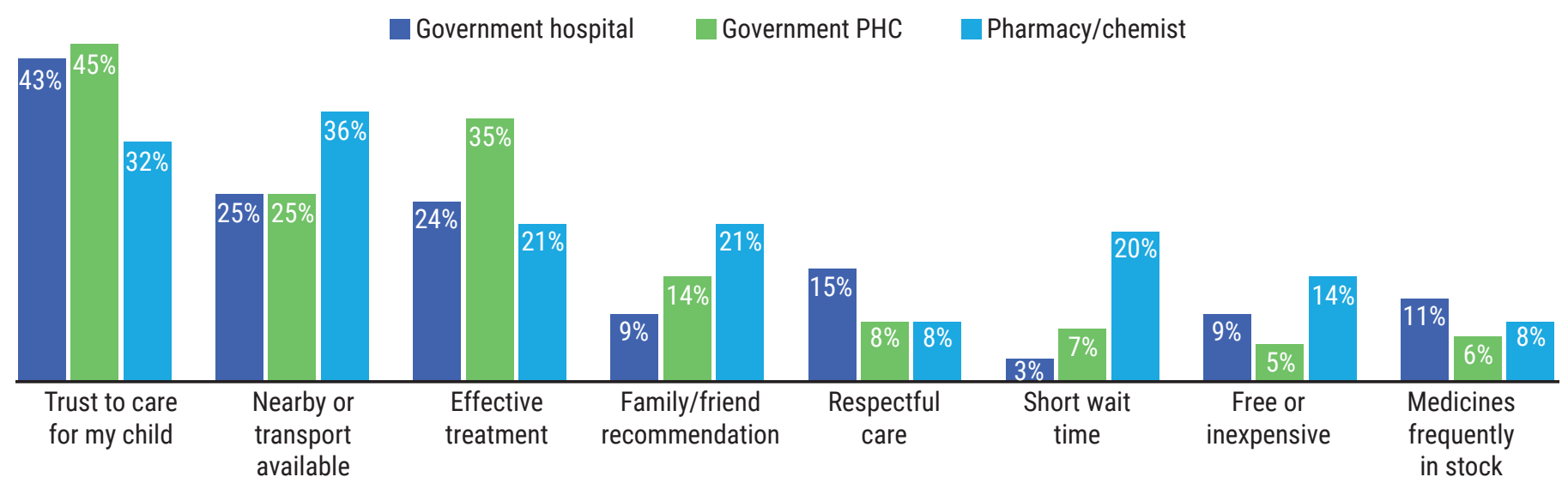

*Pharmacy/chemist was the response category used in the questionnaire and does not differentiate across the range of drug outlets from patent medicines stores, chemists, local market retailers, and other drug stores or pharmacies, which affects interpretation and conclusions drawn for this source of care. 

ing responses to knowledge questions related to malaria, pneumonia, and diarrhea

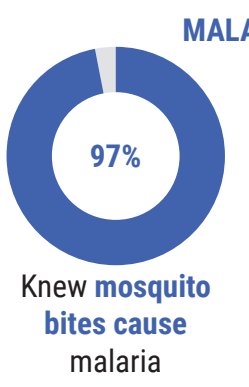

malaria

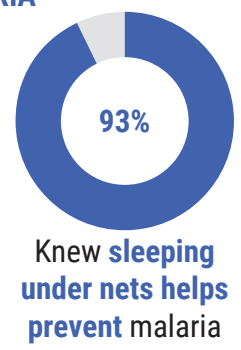

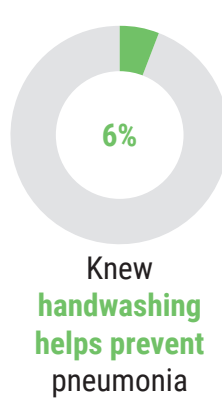

helps prevent
pneumonia

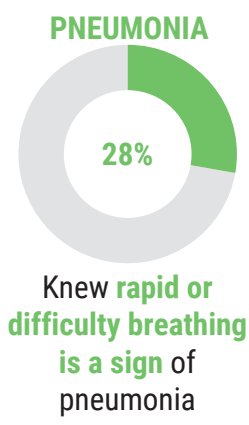

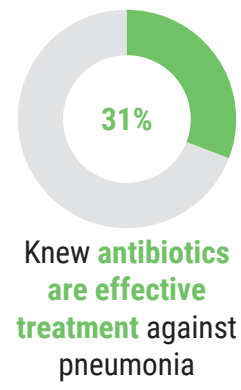

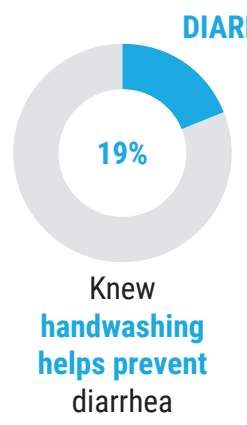

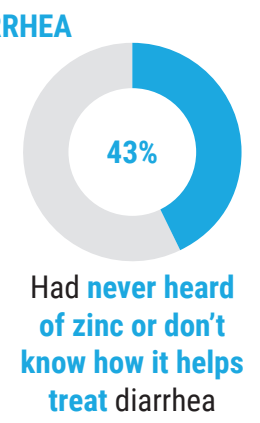

1.3-times higher likelihood of seeking care from a formal medical source for pediatric diarrhea, respiratory symptoms, or fever, respectively, than those who did not. Similarly, mothers who held this same belief were 1.6 times more likely to have their febrile child tested for malaria (Figures 5-6, next page).

- Mothers who believed a health worker is the best person to talk to for a sick child were 1.9 and 2.5 times as likely to seek formal medical care for pediatric fevers than those who did not and to give ORS and zinc treatment to children with diarrhea, respectively. Mothers who said health providers influence their decision to seek care for a sick child were 3 times as likely to treat pediatric diarrhea with ORS and zinc than those without such support.

\section{Programmatic implications}

$\checkmark \quad$ Improving perceived and actual health service quality in these communities is critical for raising formal care-seeking and appropriate treatment rates for sick children. This may require multisectoral parternships between health systems strengthening and SBC programs to improve service delivery, change provider behaviors, and improve local perceptions of health services.

\section{Knowledge, beliefs, and self-efficacy also mat- ter for sick children}

- Women who said they knew how to prepare ORS were 1.4 and 2.2 times as likely to seek formal medical care and to give ORS and zinc treatment to children with diarrhea, respectively. Women who knew that ORS and zinc were effective diarrhea treatments were 6.2 times as likely to provide them to children with diarrhea. Similarly, women who knew that antibiotics are effective treatment for pneumonia were 1.4 times as likely to seek formal care for pediatric respiratory symptoms, respectively.
- Women who believed that children can get severely ill or even die from pneumonia were 1.7 times as likely to seek formal care for pediatric respiratory symptoms than those who did not hold this belief. Women who believed that only weak children die from pneumonia were 1.4 times less likely to give antibiotics to a child with respiratory symptoms.

- Women who believed a blood test is the only way to know if a person has malaria were 2.4 times as likely to have their febrile child tested for malaria than those who did not hold that belief. Women who felt confident about convincing their husbands about seeking care for a sick child were 3.6 times as likely to have their febrile child tested for malaria than those who felt uncertain.

\section{Programmatic implications}

$\checkmark$ For pneumonia and diarrhea, SBC programs should consider raising awareness about basic prevention and treatment methods. Pneumonia risk perceptions also matter and could be an SBC message focus.

$\checkmark$ While malaria knowledge is already high, SBC programs could focus on addressing beliefs about malaria testing and women's confidence in convincing husbands to seek care for sick children. It is also possible that women who hold certain beliefs about malaria tests or have confidence to seek care are also better able to access facilities where testing is available.

$\checkmark$ Formative research is needed to better understand doubts about malaria tests and how to address them through SBC programs while also improving health services quality and access to testing. 


\section{FIGURE 5 HEALTH SERVICES PERCEPTIONS AND TREATMENT KNOWLEDGE ARE CRITICAL FOR SEEKING FORMAL MEDICAL CARE FOR A SICK CHILD}

Predicted probabilities* of seeking care from a formal medical source** for children under two years with diarrhea, fever, or respiratory symptoms by ideational factor

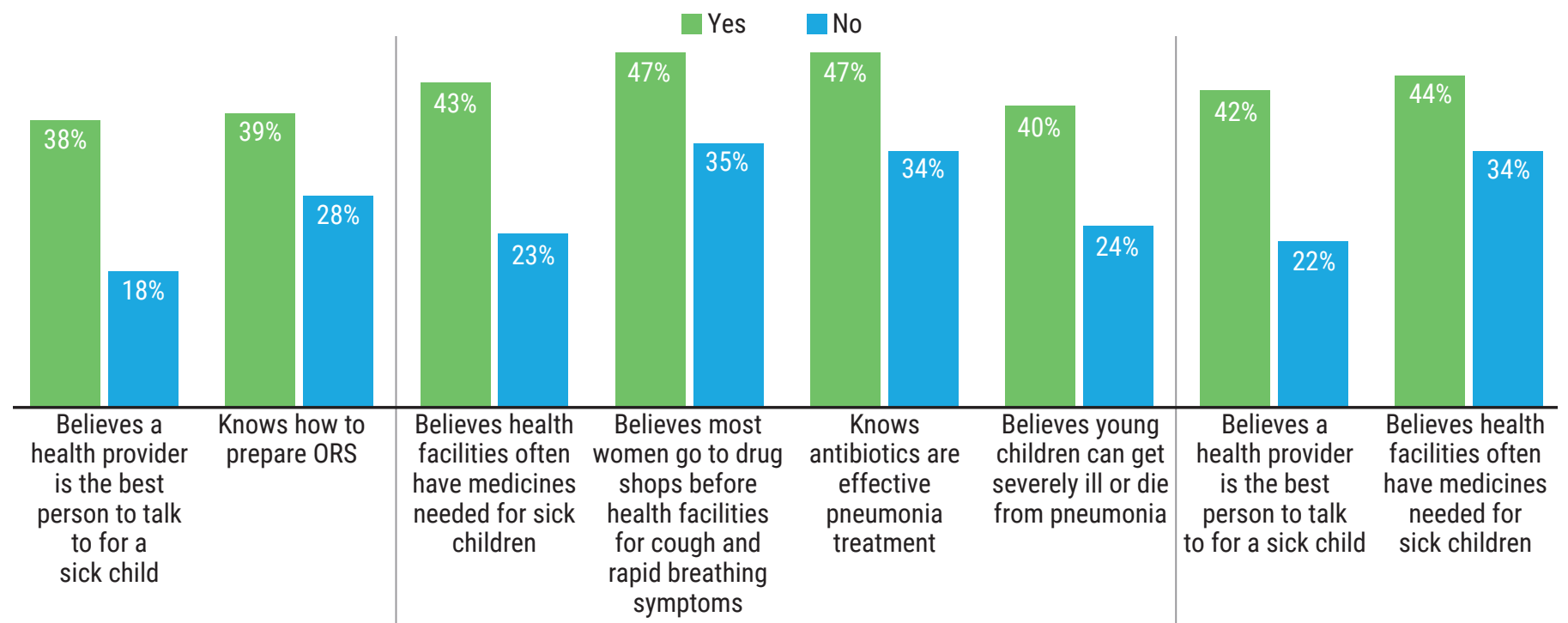

FORMAL CARE SOUGHT FOR DIARRHEA

FORMAL CARE SOUGHT FOR RESPIRATORY SYMPTOMS

FORMAL CARE SOUGHT FOR FEVER

*Predicted probabilities of formal care-seeking for sick children were derived from mixed-effects logistic regression models adjusted for child health-related ideations (Table 1) and socioeconomic characteristics including household wealth, woman's age, antenatal care attendance at least 4 times, education (woman and spouse), employment (woman and spouse), child's age, and sex. All ideational metrics presented are significant at the $<0.05$ level.

**Respiratory symptoms refer to a cough with fast or difficult breathing. Formal medical source refers to government hospital, government PHC, dispensary/health post, community health outreach post, nursing/maternity home, or private hospital/clinic. Informal sources refer to pharmacist/chemist, drug shop, traditional practitioner, family, or friend.

\section{FIGURE 6 TREATMENT KNOWLEDGE, SELF-EFFICACY AND HEALTH SERVICES PERCEPTIONS MATTER FOR APPROPRIATE DIAGNOSIS AND TREATMENT OF SICK CHILDREN}

Predicted probabilities* of ORS and zinc treatment for under-2s with diarrhea, malaria diagnosis for under-2s with fever, and antibiotic treatment for under-2s with respiratory symptoms, ${ }^{* *}$ by ideational factor

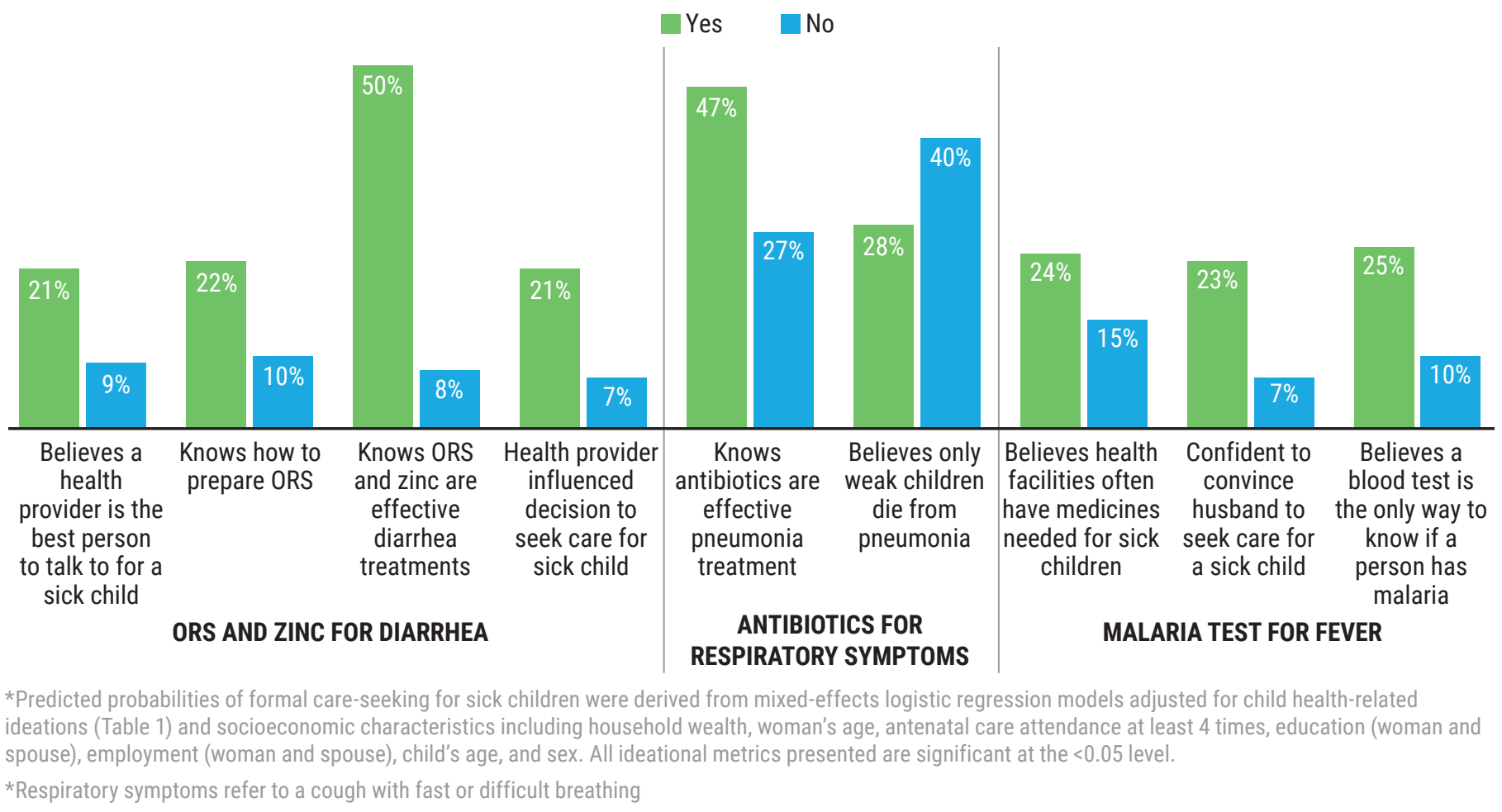




\section{Conclusions}

The results presented in this brief indicate areas where SBC programming may help improve care-seeking for sick children, diagnosis, and treatment practices in northwestern Nigeria. To this end, findings point to the most important ideations for SBC programs to address including perceived health services quality, knowledge about pneumonia and diarrhea treatment practices, and beliefs about pneumonia risks and malaria test accuracy.

While general malaria knowledge was high, knowledge of pneumonia and diarrhea prevention and treatment practices were much lower. Awareness about pneumonia and diarrhea treatment practices is a critical first step for improving sick child care for diarrhea and respiratory symptoms. SBC programs should consider focusing on dispelling these misperceptions about pneumonia risks and malaria test accuracy in order to help raise care-seeking, diagnosis and treatment rates, which can be done alongside efforts to improve health services quality and access to treatments.

Improved knowledge and beliefs about common childhood illnesses alone is not sufficient. Our results reinforce the importance of perceived (and actual) health services quality for sick child care. Women who believed health facilities frequently had medicines needed for sick children or who believed a health provider was the best person to talk to for a sick child were significantly more likely to seek care from a formal medical source for all illness symptoms, give ORS and zinc treatment for pediatric diarrhea, and have their febrile child tested for malaria. Nearby location, provider trust and perceived effectiveness of treatments were the main reasons cited by respondents for choosing the treatment location if they sought care for a sick child, underscoring desires for trusted, convenient and quality health services in the local community. Moreover, women who attended pharmacies/ chemists more often cited short wait time, lower costs, and nearby location as the reason for choosing the treatment location compared to women attending government hospitals or PHCs who instead cited provider trust and effective treatment as drivers of their choice. Finally, healthcare costs may need to be specifically addressed in health systems strengthening efforts since actual and perceived healthcare costs were a main reason for not seeking any care at all, as well as a driver for choosing pharmacies for sick child care.

\section{Annex 1: Child health-related ideational metrics}

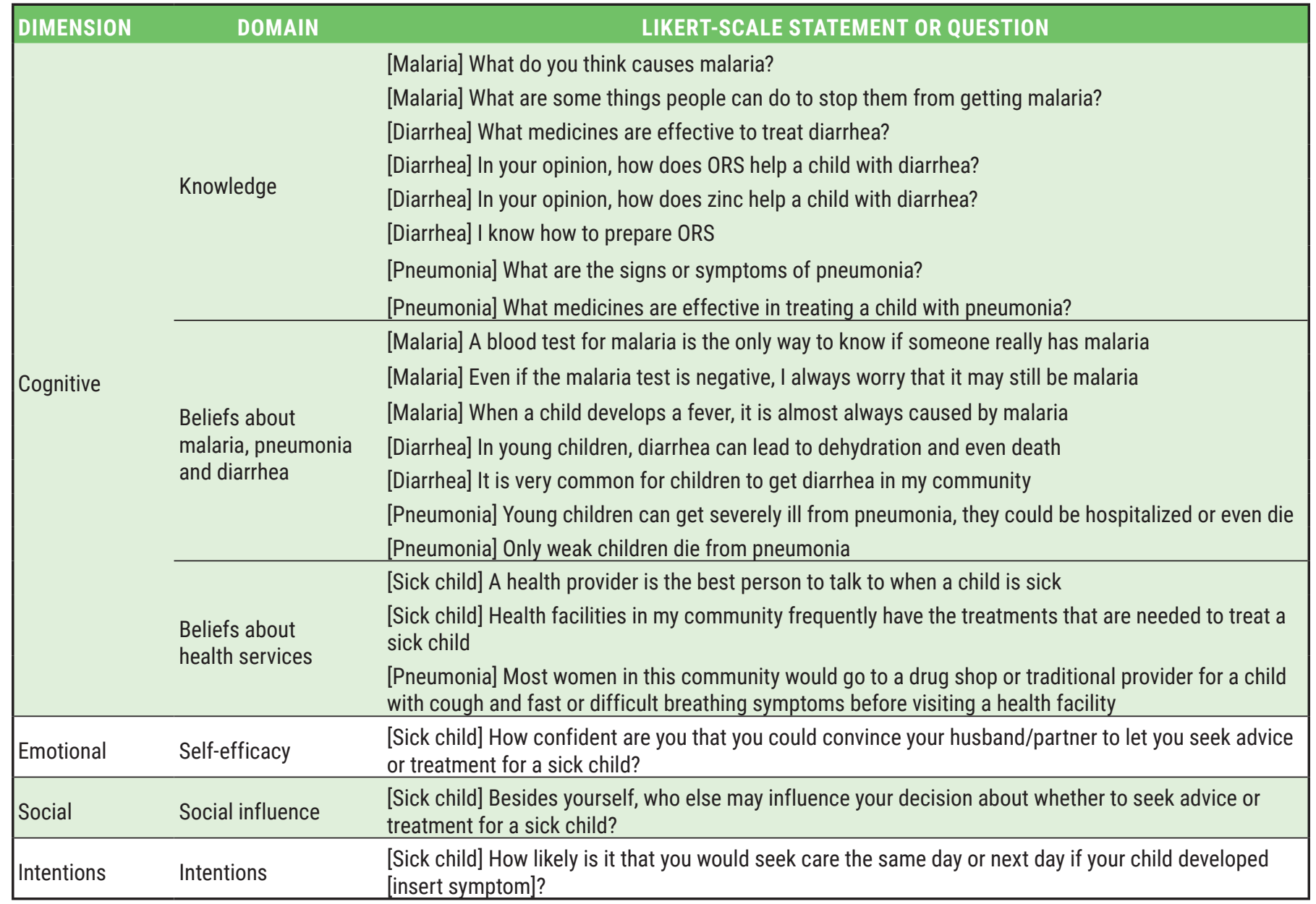




\section{References}

1. Countdown to 2015. Countdown to 2015: Maternal, Newborn \& Child Survival, The 2015 Report. Accessed at: http://countdown2030.org/documents/2015Report/Nigeria_2015.pdf

2. Gove, S. 1997. "Integrated management of childhood illness by outpatient health workers: technical basis and overview." WHO Bulletin 75(Suppl 1):7-24.

3. WHO. 2009. "Parasitological confirmation of malaria diagnosis: report of a WHO technical consultation." Geneva: WHO.

4. DHS. 2019. Nigeria Demographic and Health Survey 2018. Maryland, USA: The DHS Program.

5. Koffi, A.K. et al. 2017. "Beyond causes of death: the social determinants of mortality among children aged 1-59 months in Nigeria from 2009 to 2013," PLoS One 12(5). doi: 10.1371/journal.pone.0177025

6. Ogunrinde, O. G. et al. 2012. "Knowledge, attitude and practice of home management of childhood diarrhoea among caregivers of under-5 children with diarrhoeal disease in Northwestern Nigeria," Journal of Tropical Pediatrics 58(2): 143-146. doi: 10.1093/tropej/fmr048

7. Bedford, K. J. A. and A. B. Sharkey. 2012. "Local barriers and solutions to improve care-Seeking for childhood pneumonia, diarrhoea and malaria in Kenya, Nigeria and Niger: a qualitative study," PLoS One 9(6): e100038. doi: 10.1371/journal. pone.0100038

8. Treleavan, E. et al. 2015. "Management of paediatric illnesses by patent and proprietary medicine vendors in Nigeria," Malaria Journal 14(1): 232. doi: 10.1186/s12936-015-0747-7.

9. Kincaid, D.L. 2000. "Mass media, ideation, and behavior: a longitudinal analysis of contraceptive change in the Philippines," Communicatoin Research 27( 6): 723-763. doi: 10.1177/009365000027006003

10. Krenn, S. et al. 2014. "Using behavior change communication to lead a comprehensive family planning program: the Nigerian Urban Reproductive Health Initiative," Global Health Science and Practice 2(4):427-443. doi: 10.9745/GHSP-D-14-00009

11. Do, M. et al. 2018. "Associations between malaria-related ideational factors and care-seeking behavior for fever among. Children under five in Mali, Nigeria and Madagascar." Malaria Journal 13(1): e0191079. doi: 10.1371/journal.pone.0191079

12. Greenland, K. et al. 2017. "Disentangling the effects of multiple behavior change intervention for diarrhea control in Zambia: a theory-based process evaluation," Globalization and Health 13(1): 78. doi: 10.1186/s12992-017-0302-0

\section{Acknowledgments}

This programmatic research brief describes work led by Tulane University under Breakthrough RESEARCH. This brief and the work it describes is possible through the work and support of the Center for Research, Evaluation Resources and Development (CRERD), Breakthrough ACTION in Nigeria, Population Reference Bureau, and Population Council in Washington, DC.

\section{Suggested citation}

Breakthrough RESEARCH. 2020. "Care-seeking and treatment for sick children-Insights for improving malaria, family planning, and maternal and child health outcomes in northwestern Nigeria through social and behavior change programming," Programmatic Research Brief. Abuja, Nigeria: Population Council.

(C) 2020 The Population Council. All rights reserved.

\section{Email}

\section{BreakthroughResearch@popcouncil.org}

\section{Breakthrough RESEARCH | Population Council}

Plot 839 Idris Ibrahim Crescent | Jabi, Abuja, Nigeria +2348067787750

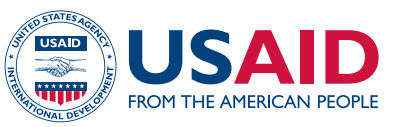

Breakthrough RESEARCH is made possible by the generous support of the American people through the United States Agency for International Development (USAID) under the terms of cooperative agreement no. AIDOAA-A-17-00018. The contents of this document are the sole responsibility of Breakthrough RESEARCH and Population Council and do not necessarily reflect the views of USAID or the United States Government.
POPULATION COUNCIL

Ideas. Evidence. Impact.

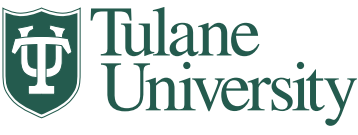

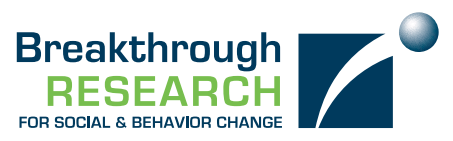

Our project turns evidence into action by providing thought provoking guidance to improve SBC policy and programming, with the goal of improving the cost-effectiveness of USAID's health and development strategies. Breakthrough RESEARCH catalyzes SBC by conducting state-of-the-art research and evaluation and promoting evidence-based solutions to improve health and development programs around the world. Breakthrough RESEARCH is a consortium led by the Population Council in partnership with Avenir Health, ideas42, Institute for Reproductive Health at Georgetown University, Population Reference Bureau, and Tulane University. 